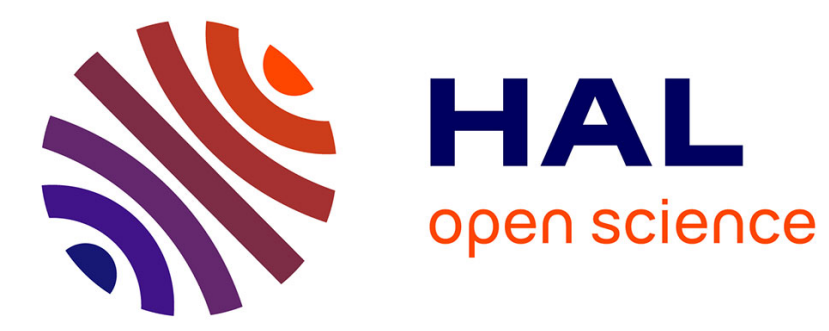

\title{
Infrared spectroscopy of molecules with nanorod arrays: a numerical study
}

\author{
C. Tardieu, G. Vincent, R. Haïdar, S. Collin
}

\section{To cite this version:}

C. Tardieu, G. Vincent, R. Haïdar, S. Collin. Infrared spectroscopy of molecules with nanorod arrays: a numerical study. Optics Letters, 2016, 41 (8), pp.1744-1747. 10.1364/OL.41.001744 . hal-01394678

\section{HAL Id: hal-01394678 \\ https://hal.science/hal-01394678}

Submitted on 9 Nov 2016

HAL is a multi-disciplinary open access archive for the deposit and dissemination of scientific research documents, whether they are published or not. The documents may come from teaching and research institutions in France or abroad, or from public or private research centers.
L'archive ouverte pluridisciplinaire HAL, est destinée au dépôt et à la diffusion de documents scientifiques de niveau recherche, publiés ou non, émanant des établissements d'enseignement et de recherche français ou étrangers, des laboratoires publics ou privés. 


\begin{tabular}{|} 
ARTICLE DE REVUE \\
Infrared spectroscopy of molecules \\
with nanorod arrays: \\
a numerical study \\
C. Tardieu, G. Vincent, R. Haìdar, \\
S. Collin (LPN-CNRS) \\
OPTICS LETTERS \\
Vol. 41, No. 8, pp. 1744-1747 \\
TP 2016-638
\end{tabular}

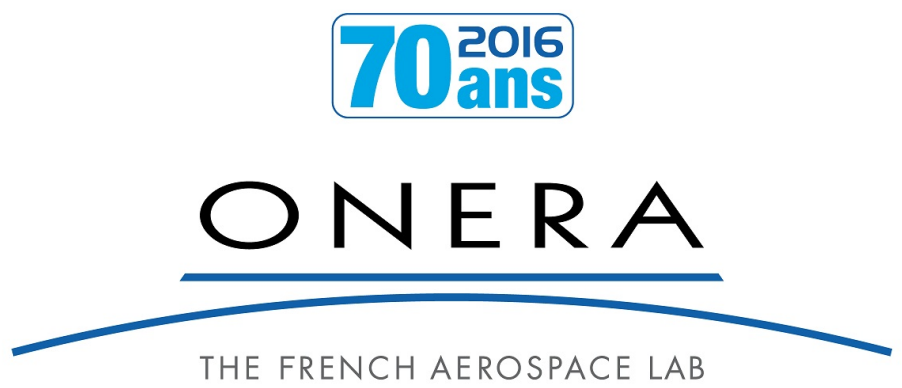





\title{
Optics Letters
}

\section{Infrared spectroscopy of molecules with nanorod arrays: a numerical study}

\author{
Clément Tardieu, ${ }^{1,2}$ Grégory Vincent, ${ }^{1}$ Riad Haïdar, ${ }^{1}$ and Stéphane Collin ${ }^{2, *}$ \\ ${ }^{1}$ ONERA - The French Aerospace Lab, Chemin de la hunière, BP 80100, F-91123 Palaiseau, France \\ ${ }^{2}$ Laboratoire de Photonique et de Nanostructures (LPN-CNRS), Route de Nozay, F-91460 Marcoussis, France \\ ${ }^{*}$ Corresponding author: stephane.collin@/pn.cnrs.fr
}

Received 11 February 2016; revised 8 March 2016; accepted 8 March 2016; posted 9 March 2016 (Doc. ID 259258); published 6 April 2016

Nanorod arrays with diameters much smaller than the wavelength exhibit sharp resonances with strong electricfield enhancement and angular dependence. They are investigated for enhanced infrared spectroscopy of molecular bonds. The molecule 3-cyanopropyldimethylchlorosilane (CS) is taken as a reference, and its complex permittivity is determined experimentally in the 3-5 $\mu \mathrm{m}$ wavelength range. When grafted on silicon nitride nanorods, we show numerically that its weak absorption bands due to chemical bond vibrations can be enhanced by several orders of magnitude compared with unstructured thin film. We propose a figure of merit (FoM) to assess the performance of this spectroscopic scheme, and we study the impact of the nanorod cross section on the FoM. (C) 2016 Optical Society of America

OCIS codes: (310.6628) Subwavelength structures, nanostructures; (300.6340) Spectroscopy, infrared; (280.1415) Biological sensing and sensors; (300.6390) Spectroscopy, molecular.

http://dx.doi.org/10.1364/OL.41.001744

Molecular spectroscopy is based on the detection of specific rotational and vibrational states of molecular bonds. Most of them are in the infrared spectral domain and can be detected by direct and indirect optical methods. Raman spectroscopy is a widely used indirect technique [1] based on inelastic scattering of visible light. Vibrational states are associated with the spectral shift of scattered photons. The weakness of the Raman signals is counterbalanced by the availability of powerful laser light sources in the visible and can be partly overcome by surfaceenhanced Raman spectroscopy. On the other hand, the specific vibrational states of molecular bonds can be directly measured in the infrared (IR) domain by absorption spectroscopy. Actually, Raman spectroscopy and IR spectroscopy are complementary methods and can detect different vibrational states according to the symmetry properties of the molecules [2].

Applications of absorption spectroscopy to low quantities of molecules are hindered by the very weak absorption of molecule bonds in the infrared and by the low brightness of thermal light sources. It can be enhanced by surface-enhanced infrared absorption (SEIRA). Many SEIRA antennas are based on rod-shaped metallic structures [3,4]. Plasmonic resonances in the infrared induce a strong intensity enhancement localized in the vicinity of the nanostructures. The vibrational signals of a small number of molecules adsorbed on the metal can be enhanced by several orders of magnitude if they coincide with the resonance wavelength [4]. Alternatively, other designs, such as bowtie antennas [5], are used to improve the confinement of the near-field intensity, yet with a weaker enhancement. In references [6,7], the authors investigate the fan-shaped antenna that combines the advantages of the first two designs, but the enhancement is spectrally limited by the resonance bandwidth. These antennas can hardly be used for the detection of several vibrational modes extending over a large spectral range.

Here, we propose a different scheme for enhanced IR absorption spectroscopy of a thin layer of molecules. It is based on free-standing dielectric nanorod arrays with diameters much smaller than the wavelength [8]. For transverse electric (TE) polarized light, such arrays exhibit sharp resonances with strong electric-field enhancement around the nanorods. Moreover, the resonance wavelength can be tuned over a large spectral range with the incidence angle. We have chosen the 3-cyanopropyldimethylchlorosilane (CS) molecule as a reference for this numerical study. Its complex dielectric permittivity has been experimentally determined and exhibits weak infrared absorption bands in the 3-5 $\mu \mathrm{m}$ range. We investigate nanorod arrays with various cross sections, covered with a monolayer of CS molecules. We show that the weak absorption bands of CS can be enhanced by several orders of magnitude. We propose a figure of merit (FoM) to asset the performance of this spectroscopic scheme, and we compare our numerical results with SEIRA experiments.

The structure consists of a freestanding subwavelength nanorod array made of dielectric material with a period $P=$ $3 \mu \mathrm{m}$ (see Fig. 1 [inset]). We first consider silicon nitride (SiN) nanorods with a section $H \times W=300 \mathrm{~nm} \times 300 \mathrm{~nm}$ and a refractive index $n=2$ [9]. Each individual nanorod acts as a nonresonant scatterer with a weak scattering cross section due to its small width $(<\lambda / 10)$ [10]. The collective excitation of regularly spaced nanorods by a plane wave results in a constructive interference. Numerical examples are shown in Fig. 1. They are based on rigorous coupled-wave analysis calculations $[11,12]$. Absolute reflection spectra, i.e., reflection intensity 


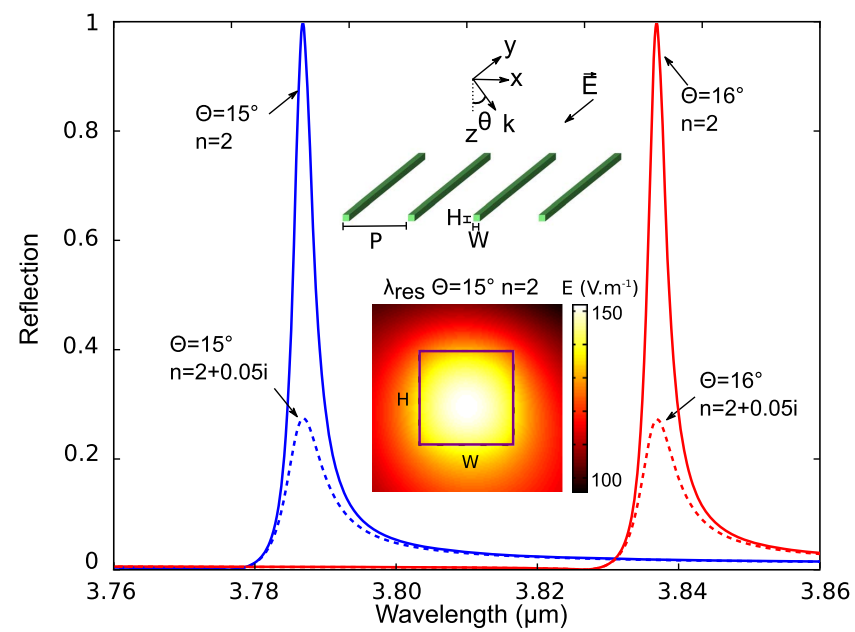

Fig. 1. Calculated reflection spectra of nanorods with a period $P=$ $3 \mu \mathrm{m}$ and a rod section $H \times W=300 \mathrm{~nm} \times 300 \mathrm{~nm}$ for TEpolarized plane waves at $15^{\circ}$ (blue) and $16^{\circ}$ (red) with $n=2$ (solid lines) and $n=2+0.05 i$ (dashed lines). Inset: schematic of the nanorod array (top); calculated map of electric field intensity at the maximum of reflection (bottom, linear color scale).

normalized to the incident plane wave intensity, are plotted for transverse electric (TE) polarized plane wave at $15^{\circ}$ (blue) and $16^{\circ}$ (red). In the far-field, reflection spectra exhibit a sharp resonance due to this multiple coherent scattering [13]. Optical resonance leads to perfect reflection at $\lambda_{\text {res }}$, and the resonance wavelength increases with the angle of incidence. The map of the electric field intensity at $\lambda_{\text {res }}$ and $\theta=15^{\circ}$ is also shown in Fig. 1. The electric field is strongly enhanced in the vicinity of the rods.

We now consider the same structure with weak optical losses $(n=2+0.05 i)$. The numerical results are shown in Fig. 1 (dashed lines). The resonance wavelength and bandwidth are nearly unchanged, but the reflection maximum drops drastically. The spectroscopic detection method described in this Letter is based on the perturbation of the resonant reflection due to the presence of an absorbing monolayer of molecules grafted on the rods. Infrared absorption due to molecular bonds will be enhanced by the strong electric field and detected through the variations of the reflection maximum. The nanorod cross section is smaller, the resonance is narrower, and the electric field is higher. In Table 1, we report the FWHM for various cross sections of nanorods at normal incidence.

The molecule to be detected must be carefully chosen: adsorption of thiols is possible on nanoantennas made of gold but not on silicon nitride. Here, the CS molecule has been selected as a model molecule because of (1) its affinity with silicon and (2) several molecular bond vibrations in the mid-infrared. Its permittivity was determined experimentally by Fourier transform infrared (FTIR) spectroscopy with a resolution of $4 \mathrm{~cm}^{-1}$. A fluidic chamber with $\mathrm{CaF}_{2}$ windows and of various

Table 1. FWHM of Different Sections of Cross Nanorods at $0^{\circ}$

\begin{tabular}{lccccc}
\hline Section $(\mathbf{n m})$ & $\mathbf{5 0 0}$ & $\mathbf{3 0 0}$ & $\mathbf{2 0 0}$ & $\mathbf{1 0 0}$ & $\mathbf{5 0}$ \\
\hline FWHM $(\mathrm{nm})$ & 185.7 & 20.3 & 2.0 & $3 \cdot 10^{-2}$ & $4 \cdot 10^{-4}$ \\
\hline
\end{tabular}

well-controlled thicknesses ( 15 and $25 \mu \mathrm{m}$ ) was filled with the CS molecule in a pure liquid solution.

In the 3-5 $\mu \mathrm{m}$ spectral range, the dielectric permittivity of $\varepsilon_{\mathrm{CS}}$ is modeled by a sum of $\varepsilon_{\infty}$ and five Lorentz oscillators: $\varepsilon_{\mathrm{CS}}=\varepsilon_{\infty}+\Sigma \frac{\Delta \varepsilon_{i} \omega_{o i}^{2}}{\omega_{o i}^{2}-\omega^{2}-i \gamma_{i} \omega}$ where $\Delta \varepsilon_{i}, \omega_{o i}$, and $\gamma_{i}$ are the strength, frequency, and linewidth of the i-th Lorentz oscillator, respectively, and $\varepsilon_{\infty}$ is the high-frequency permittivity. These oscillators are introduced in order to model absorption bands due to the vibration of $\mathrm{CH}_{2}$ and $\mathrm{CH}_{3}$ bonds near $3.4 \mu \mathrm{m}$ and $C \equiv N$ bond at $4.45 \mu \mathrm{m}$. Transmission measurements were used to fit all the parameters: $\varepsilon_{\infty}=2.09, \Delta \varepsilon_{i}=[36,50,27$, $55,62], \quad \omega_{o i}\left(\mathrm{~cm}^{-1}\right)=[2247,2880,2905,2936,2962], \quad$ and $\gamma_{i}\left(\mathrm{~cm}^{-1}\right)=[8.9,29.9,21.8,26.4,26.5]$. The real and imaginary parts of $\varepsilon_{\mathrm{CS}}$ are plotted in Fig. 2(a).

A monolayer of self-assembled CS molecules adsorbed on nanorods is modeled by a uniform layer of thickness $t_{\mathrm{CS}}=$ $2.8 \mathrm{~nm}$, covering each side of the rods [sketch in Fig. 2(b)]. Figure 2(c) shows numerical results for a grating with period $P=3 \mu \mathrm{m}$ and section $H \times W=100 \mathrm{~nm} \times 100 \mathrm{~nm}$. Numerous reflection spectra are calculated for various incidence angles between $0^{\circ}$ and $30^{\circ}$ [only a few spectra are shown in Fig. 2(c)]. As a result, the resonance wavelength scans a spectral range from $3 \mu \mathrm{m}$ to $4.5 \mu \mathrm{m}$. As compared with Fig. 1, resonances are much narrower due to the smaller cross-section area. The reflection maximum is strongly impacted by the absorption bands of the CS molecules and drops down to $60 \%$. The envelop of the reflection maxima $\left[R_{\max }\right.$, dashed line in Fig. 2(c)] is also plotted. It nicely reflects the imaginary part of $\varepsilon_{\mathrm{CS}}$ and provides the characteristic spectrum of the molecular vibrations of the thin layer of CS molecules.

The electric field enhancement increases with smaller nanorod cross sections [8], leading to a stronger impact of the CS layer on the reflection spectra. In Fig. 3, we present $R_{\max }$

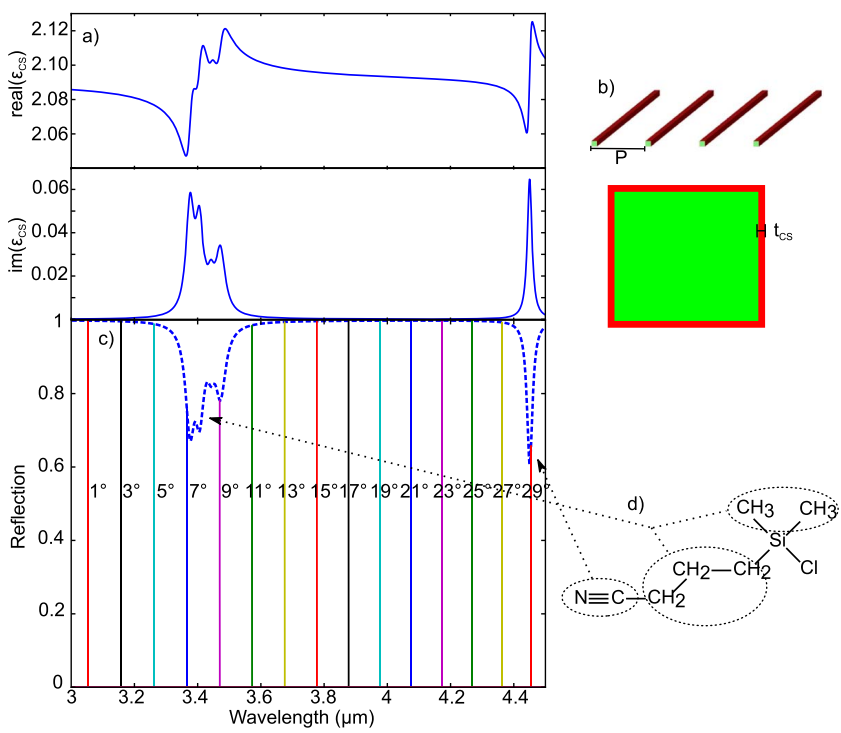

Fig. 2. (a) Real and imaginary parts of the permittivity of the (3-cyanopropyl)dimethylchlorosilane (CS). (b) Sketch of nanorods encapsulated with a layer of CS (geometric scale is not respected). (c) Calculated reflection spectra of nanorods covered with a $2.8 \mathrm{~nm}$ layer of CS (period $P=3 \mu \mathrm{m}$, section $H \times W=100 \mathrm{~nm} \times 100 \mathrm{~nm})$ for TE-polarized plane waves at different incidence angles. Dashed line: envelope of the reflection maxima. (d) Diagram of the molecule CS. 
envelopes for various rod sections compatible with the nanofabrication process $[14,15]$. Narrow rods with a section of $50 \mathrm{~nm} \times 50 \mathrm{~nm}$ allow us to reach a reflection intensity down to $10 \%$ for the $C \equiv N$ bond resonance, whereas with a section of $200 \mathrm{~nm} \times 200 \mathrm{~nm}$ intensity only drops down to $93 \%$ at the same wavelength.

Plasmonic biosensors are based on the spectral shift of optical resonances due to the presence of molecules at the surface of a metal [16]. Their figure of merit (FoM) can be defined by the ratio between the relative intensity change (at a fixed wavelength) and the (real part) of the refractive index change induced by the molecules [17]. Here, we adapt this definition for infrared absorption spectroscopy of molecules enhanced by nanorods or nanoantennas. The FoM is defined as the ratio of the reflected intensity $\Delta R$ and the imaginary part of the refractive index of the molecule $\Delta k$ :

$$
\mathrm{FoM}=\frac{\Delta R}{\Delta k},
$$

where $\Delta R$ and $\Delta k$ are the differences in reflection intensity and imaginary part of the refractive index of the molecule, respectively, for a component with and without molecules.

For the $C \equiv N$ bond $(\lambda=4.45 \mu \mathrm{m}), \Delta k_{\mathrm{CS}}=2.2 \cdot 10^{-2}$. We evaluate $\Delta R$ for different nanorod cross sections and calculate the FoM in each case. The results are reported in Table 2. This table also gives the absorption of an unstructured thin film of CS molecules. The thickness of the thin film is calculated so that the amount of CS is the same as for nanostructures. They range from FoM $=0.2$ for $500 \mathrm{~nm} \times 500 \mathrm{~nm}$ cross sections to FoM $=40.9$ for $50 \mathrm{~nm} \times 50 \mathrm{~nm}$ cross sections. While the perimeter decreases by a factor of 10 , the FoM increases by a factor of approximately 200 .

In Table 3, we report the FoM calculated for the study described in [4], involving the $\mathrm{CH}_{2}$ bonds of octadecanthiol (ODT) molecule on gold nanoantennas: FoM = 1.9. In order to compare the FoM of nanoantennas and nanorods systems, we achieved new calculations assuming a monolayer of ODT is grafted on nanorods (in fact, thiols molecules cannot be adsorbed on silicon nitride). The dielectric permittivity of ODT has been taken from [18]. A bond resonance occurs at $\lambda=3.42 \mu \mathrm{m}$, with

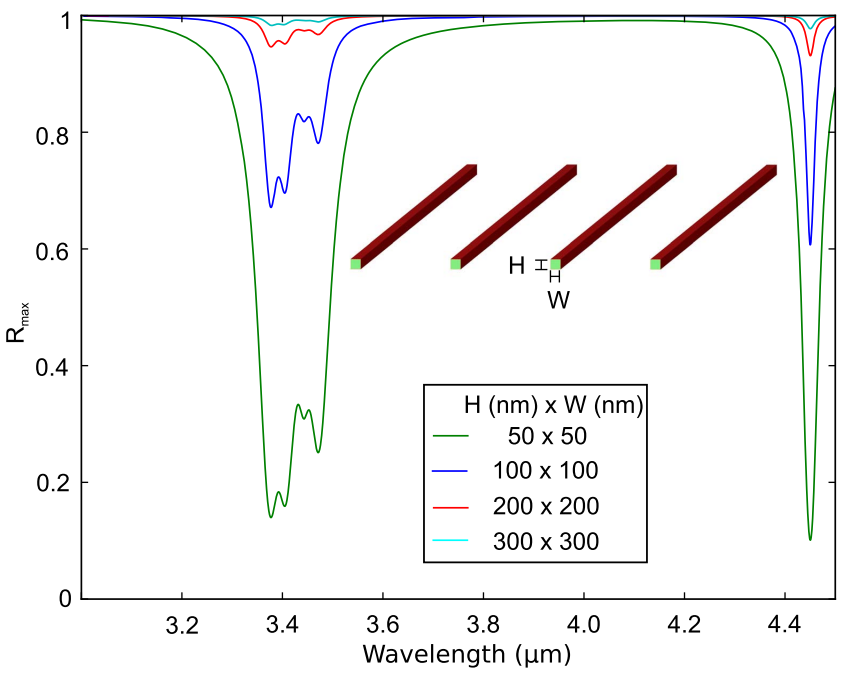

Fig. 3. Envelope of the reflection maximum calculated for nanorods covered with a $2.8 \mathrm{~nm}$ layer of CS molecules, with different cross sections in TE-polarized plane wave.
Table 2. Figure of Merit for Different Nanorod Cross Sections with a $2.8 \mathrm{~nm}$ Layer of CS at $4.45 \mu \mathrm{m}(C \equiv N$ bond) and Absorption of Equivalent Unstructured Layer

$\mathrm{CS}: \Delta k=2.2 \cdot 10^{-2}$

\begin{tabular}{lccc}
\hline Structure & FoM & $\boldsymbol{\Delta} \boldsymbol{R}$ & $\begin{array}{c}\text { Equivalent Layer } \\
\text { Absorption }\end{array}$ \\
\hline Nanorods 50 nm & 40.9 & $90 \%$ & $1.2 \cdot 10^{-3} \%$ \\
Nanorods 100 nm & 17.7 & $39.3 \%$ & $2.3 \cdot 10^{-3} \%$ \\
Nanorods 200 nm & 3.1 & $6.9 \%$ & $4.7 \cdot 10^{-3} \%$ \\
Nanorods 300 nm & 1 & $2.3 \%$ & $7.1 \cdot 10^{-3} \%$ \\
Nanorods 500 nm & 0.2 & $0.48 \%$ & $1.2 \cdot 10^{-2} \%$ \\
\hline
\end{tabular}

Table 3. Figure of Merit for Different Sections of Nanorods with a $2.5 \mathrm{~nm}$ Layer of ODT at $3.42 \mu \mathrm{m}$ ( $\mathrm{CH}_{2}$ Bond)

\begin{tabular}{lcc}
\hline & ODT $: \boldsymbol{\Delta} \boldsymbol{k}=\mathbf{8 . 4} \cdot \mathbf{1 0}^{\mathbf{- 3}}$ & \\
\hline Structure & $\mathbf{F o M}$ & $\boldsymbol{\Delta} \boldsymbol{R}$ \\
\hline Nanoantennas [4] & 1.9 & $1.6 \%$ \\
Nanorods 50 nm & 74.2 & $62.3 \%$ \\
Nanorods 100 nm & 17.4 & $14.6 \%$ \\
Nanorods 200 nm & 2.4 & $2.0 \%$ \\
Nanorods 300 nm & 0.7 & $0.6 \%$ \\
Nanorods 500 nm & 0.1 & $0.12 \%$ \\
\hline
\end{tabular}

a corresponding refractive index of $n_{\mathrm{ODT}}=1.41+8.4 i \cdot 10^{-3}$. The thickness of the self-assembled monolayer is $t_{\mathrm{ODT}}=$ $2.5 \mathrm{~nm}$. Table 3 shows that the FoM obtained with nanoantennas and nanorods are comparable for $200 \mathrm{~nm} \times 200 \mathrm{~nm}$ cross sections. However, a reduction of the nanorod section allows us to drastically increase the value of the FoM, which reaches $\mathrm{FoM}=74.2$ for $50 \mathrm{~nm} \times 50 \mathrm{~nm}$ section.

In summary, we have numerically investigated a new strategy for infrared spectroscopy of a tiny layer of molecules. It is based on optical resonances in freestanding nanorods. The farfield optical response of the system is strongly modified by the presence of molecules grafted on the rods. Optical absorption due to molecular bond vibrations is enhanced by the strong electric-field intensity in the vicinity of the nanorods. In addition, the angular dependency of the optical resonances allows us to scan a wide spectral range. The envelope of reflection spectra reflects the absorption spectrum of the bond vibrations of the molecule. Numerical calculations have been achieved on nanorods made of silicon nitride and (3-cyanopropyl) dimethylchlorosilane molecules. We have proposed an FoM in order to assess the performances of this spectroscopy scheme. In the case of $C \equiv N$ bonds, the smallest section $(50 \mathrm{~nm} \times 50 \mathrm{~nm})$ leads to a high contrast in the reflected intensity change (90\%) and an FoM as high as 40.9. Nanorods were compared with previous SEIRA experiments achieved with gold nanoantennas and octadecanthiol molecules. One of the advantages in the use of transparent nanorods stands in the fact that no energy is lost in resonator, contrary to metallic nanostructures. Hence, the quality factor $Q$ of the resonance and, thus, field enhancement can be very high: up to $Q \approx 10^{6}$ for $50 \mathrm{~nm} \times$ $50 \mathrm{~nm}$ geometry. Higher FoM are obtained for nanorod sections smaller than $200 \mathrm{~nm} \times 200 \mathrm{~nm}$. The spectral tunability of nanorods provides an additional advantage for infrared molecular spectroscopy on a wide spectral range. 


\section{REFERENCES}

1. M. Moskovits, J. Raman Spectrosc. 36, 485 (2005).

2. B. Schrader, Infrared and Raman Spectroscopy Methods and Applications (Wiley, 2008).

3. R. Adato and H. Altug, Nat. Commun. 4, 2154 (2013).

4. F. Neubrech, A. Pucci, T. W. Cornelius, S. Karim, A. Garcia-Etxarri, and J. Aizpurua, Phys. Rev. Lett. 101, 157403 (2008).

5. N. Yu, E. Cubukcu, L. Diehl, D. Bour, S. Corzine, J. Zhu, G. Höfler, K. B. Crozier, and F. Capasso, Opt. Express 15, 13272 (2007).

6. D. Wang, T. Yang, and K. B. Crozier, Opt. Express 18, 10388 (2010).

7. L. V. Brown, X. Yang, K. Zhao, B. Y. Zheng, P. Nordlander, and N. J. Halas, Nano Lett. 15, 1272 (2015).

8. P. Guenuche, G. Vincent, M. Laroche, N. Bardou, R. Haïdar, J. Pelouard, and S. Collin, Phys. Rev. Lett. 109, 143903 (2012).

9. R. Chow, W. A. Landford, W. Ke-Ming, and R. S. Rosler, J. Appl. Phys. 53, 5630 (1982).
10. R. Gómez-Medina, M. Laroche, and J. J. Saenz, Opt. Express 14, 3730 (2006).

11. J. P. Hugonin and P. Lalanne, Reticolo Software for Grating Analysis (Institut d'Optique, 2005).

12. M. G. Moharam, E. B. Grann, D. A. Pommet, and T. K. Gaylord, J. Opt. Soc. Am. A 12, 1068 (1995).

13. M. Laroche, S. Albaladejo, R. Carminati, and J. J. Saenz, Opt. Lett. 32 , 2762 (2007).

14. G. Vincent, S. Collin, N. Bardou, J. Pelouard, and R. Haïdar, J. Vac. Sci. Technol. B 26, 1852 (2008).

15. T. A. Savas, S. N. Shah, M. L. Shattenburg, J. M. Carter, and H. I. Smith, J. Vac. Sci. Technol. B 13, 2732 (1995).

16. C. Valsecchi and A. G. Brolo, Langmuir 29, 5638 (2013).

17. A. Cattoni, P. Ghenuche, A. Haghiri-Gosnet, D. Decanini, J. Chen, J. Pelouard, and S. Collin, Nano Lett. 11, 3557 (2011).

18. A. Garcia-Etxarri, Modelization of Plasmonic Nanoantennas for Optical Microscopy and Surface Enhanced Spectroscopy (Universidad del pais Vasco, 2010). 

Office National d'Études et de Recherches Aérospatiales Chemin de la Hunière et des Joncherettes - BP 80100 91123 PALAISEAU Cedex

Tél. : +33180386060-Fax : +33180386510 http://www.onera.fr 